Archives de sciences sociales des religions

113 | janvier-mars 2001

Varia

\title{
La Place et le rôle des femmes dans les Églises réformées
}

Raymond A. Mentzer

\section{(2) OpenEdition}

12 Journals

Édition électronique

URL : http://journals.openedition.org/assr/20192

DOI : 10.4000/assr.20192

ISSN : $1777-5825$

Éditeur

Éditions de l'EHESS

Édition imprimée

Date de publication : 1 avril 2001

Pagination : 119-132

ISBN : 2-222-96701-5

ISSN : 0335-5985

Référence électronique

Raymond A. Mentzer, "La Place et le rôle des femmes dans les Églises réformées ", Archives de sciences sociales des religions [En ligne], 113 | janvier-mars 2001, mis en ligne le 19 août 2009,

consulté le 19 avril 2019. URL : http://journals.openedition.org/assr/20192 ; DOI : 10.4000/assr.20192

Ce document a été généré automatiquement le 19 avril 2019

(c) Archives de sciences sociales des religions 


\title{
La Place et le rôle des femmes dans les Églises réformées
}

\author{
Raymond A. Mentzer
}

1 La Réforme, tant protestante que catholique, a profondément modifié la condition des femmes au cours des XVI ${ }^{e}$ et XVII ${ }^{e}$ siècles. C'est certain. Mais on est moins assuré pour définir la nature et la signification de ces changements. La question doit cependant être posée. Quelles nouveautés la Réforme a-t-elle apportées dans la vie des femmes ? De quoi s'agit-il exactement et comment est-il possible d'en évaluer les effets?

2 Les changements peuvent concerner le statut conjugal et familial de la femme et son rôle dans la congrégation. Dans tous les cas, les réponses devront être d'autant plus nuancées que la situation des réformés dans le Royaume a changé au cours des années, avant et après l'Édit de Nantes (1598), avant et après son application "à la rigueur" (1660). De plus les situations locales sont contrastées entre la France du Nord et celle du Midi, entre les lieux où les réformés sont majoritaires ou très nombreux et ceux où ils sont très disséminés, leurs conditions sociales définissant en outre des variables supplémentaires. Autre raison qui invite à la prudence, les sources auxquelles les historiens ont recours sont lacunaires, qu'il s'agisse des actes notariés ou des registres des consistoires. Ce sont ceux de ces registres consistoriaux qui ont été préservés, et dont nous achevons d'établir un inventaire, que nous avons ouverts pour rédiger ces pages.

3 Le contrôle de l'observation de là discipline, la discipline du mariage notamment, était en effet exercé par un consistoire formé au sein de chaque Église locale. Présidé par le pasteur, le consistoire réunit les anciens, ou surveillants, et les diacres. Il est compétent, dans le respect des décisions prises par les synodes nationaux et provinciaux, en matière d'administration ecclésiastique, de liturgie, d'action charitable et de contrôle moral. Le consistoire - composé exclusivement d'hommes - convoque les fidèles signalés comme défaillants. Il les exhorte à la repentance et à la réconciliation. En cas de refus, il peut prononcer une suspension de la cène, ou même une excommunication, ou encore adresser les délinquants à la justice du magistrat. Les membres du consistoire - «élus ", c'est-à-dire cooptés - ne se soustraient pas à la censure. 
4 On a estimé de ce fait qu'il faut considérer le consistoire comme un service obligatoire d'aide et de conseil chargé, entre autres tâches, de réguler le mariage, de renforcer les liens entre époux, et de résoudre les conflits intra-familiaux ${ }^{1}$. De nombreux registres d'actes de consistoires réformés des $\mathrm{XVI}^{\mathrm{e}}$ et XVII ${ }^{\mathrm{e}}$ siècles ont déjà été analysés ou publiés. Nos recherches personnelles nous ont ouvert l'accès à plusieurs dizaines de registres, ou fragments de registres, jusque-là ignorés. Elles nous ont mis en présence de multiples informations qui fondent nos observations et interprétations.

5 C'est donc à partir de ces sources que nous rappellerons et critiquerons les observations de deux spécialistes bien connus dans le monde anglo-américain: l'américaine Natalie Davis et l'australienne Lyndal Roper.

\section{Bilan historiographique}

6 Natalie Davies et certains historiens, dans des études publiées pour l'essentiel dans la période des premières recherches sur ce sujet, ont souligné les aspects positifs de la Réforme pour la condition des femmes ${ }^{2}$. Dans un long article «Les Huguenotes ", écrit il y a plus de vingt-cinq ans, Natalie Davis a insisté sur cette amélioration de la condition féminine par la Réforme protestante ${ }^{3}$. Elle indique, par exemple, que les femmes protestantes savaient plus fréquemment lire et écrire que leurs sœurs catholiques. La femme protestante s'était en effet engagée, selon Davis, dans la tâche sacrée et grave de la lecture et de la discussion de l'Écriture sainte, ce qui au Moyen-Âge était exclusivement réservé aux hommes.

7 La femme dans l'analyse de Davis fut également active dans tous les moments de la Réforme en France. Elle manifesta contre l'idolâtrie papiste, et plusieurs figurent parmi les martyrs célébrés dans l'Histoire des martyrs de Jean Crespin 4 . La nouvelle liturgie était en langue vulgaire - la langue des femmes et du menu peuple - et par ce moyen elle autorisait la participation féminine. Pour rester dans ce domaine, lieu de la compétence masculine au Moyen-Âge, la femme protestante, autant que son mari, chantait les psaumes chaque dimanche au temple. Elle n'était plus exclue complètement de la participation à la liturgie.

8 Le couple ne projetait certes pas une relation de rapports d'égal à égal, mais il en allait désormais mieux qu'avant. Le pasteur est marié, et sa femme désignée comme l'épouse digne du ministre de la Parole de Dieu. Enfin, les protestants, ont souligné l'importance, dans le mariage, de l'amour et de l'amitié - une situation dont la femme profita. Ainsi, en divers domaines, la condition des femmes - qui, au Moyen-Âge étaient totalement subordonnées à l'homme - se serait améliorée chez les protestants. Élevée à un rang proche de celui du mari, elle exerce une influence croissante sur les décisions prises au sein du couple et dans bien d'autres aspects de la vie conjugale. Pour Davis la femme protestante devient une personne indépendante, dotée de pouvoir, et moderne.

D'autres spécialistes ont montré moins d'enthousiasme quant aux effets de la Réforme pour les femmes. Ils estiment en effet que la Réforme a renforcé le patriarcat. Lyndal Roper dans son livre sur le protestantisme à Augsbourg, The Holy Household: Women and Morals in Reformation Augsburg ${ }^{5}$, souligne que la Réforme, qu'il s'agisse de foi religieuse ou des répercussions sociales, reste fondamentalement une théologie sexuellement marquée. L'apport des réformateurs serait donc, en la matière, profondément ambigu. Le protestantisme selon Roper peut amener à la promotion d'une pratique pieuse féminine 
autant qu'à un patriarcat renouvelé. C'est là contredire le point de vue courant, pour lequel le protestantisme est implicitement associé aux forces progressistes, individualistes et modernes ${ }^{6}$. Au moment de l'institutionnalisation de la Réforme, les valeurs du moralisme protestant auraient été exploitées par une tradition conservatrice plus ancienne, qui a défini la femme par la soumission au mari. La Réforme, aux dires de Roper, a conduit à la domestication du clergé, mais beaucoup plus importante serait la domestication de la femme ainsi placée sous l'autorité de son mari. La Réforme aurait donc réitéré un message conservateur à propos de la régulation du rôle féminin dans le mariage et dans la maisonnée. Là aurait été la clé du succès de la Réforme, en particulier dans les milieux urbains. Les rapports sociaux entre les genres loin de représenter un point secondaire de la Réforme, en constituaient l'essentiel.

Dans son histoire de la Réforme à Augsbourg, Roper multiplie des arguments en faveur de cette thèse. Le contrôle des femmes et le contrôle des mœurs y vont de pair. Toute femme indépendante a dû se placer sous une autorité masculine et patriarcale. À Augsbourg, on a fermé les couvents des femmes aussi bien que les bordels municipaux. La nonne et la prostituée représentaient toutes deux des menaces parce qu'elles étaient des femmes indépendantes, étrangères au système hiérarchique du mariage, de la famille et de la maisonnée. L'abbesse médiévale avait exercé, il est vrai, un pouvoir immense dans les domaines économique, social et parfois politique. La prostituée pour sa part avait le contrôle de sa propre sexualité. Les consuls municipaux ont donc donné les unes et les autres - les nonnes et les prostituées - en mariage aux jeunes hommes d'Augsbourg. Par ce moyen, leur sexualité était domestiquée dans le cadre de la sainte famille, expression d'un ordre moral qui était aussi un ordre masculin.

11 Les protestants ont aussi re-défini le mariage. Le mariage restait bien sûr l'institution morale, juridique et sociale au sein de laquelle on met au monde et prend soin des enfants, cette nécessaire génération montante qui assurerait la survie et le développement de la famille, son nom et sa puissance. Mais à partir de la Réforme, le mariage et la sexualité ont été régulés avec précision. Les cérémonies de mariage, les obligations légales auxquelles se conformer au préalable, les moyens de déterminer et de résoudre les conflits conjugaux furent l'objet d'une surveillance sévère exercée par les autorités ecclésiastiques et les gouvernements municipaux. Pour la femme protestante cette discipline morale conduisait à une domination masculine. Malheureusement, Roper a peu examiné l'ensemble de l'espace où les Augsbourgeoises ont pu pratiquer la piété réformée, et elle laisse entendre qu'il s'agissait d'une pratique privée dans le cadre de la « sainte » famille.

12 Cette double dynamique - c'est-à-dire, le contrôle de la femme protestante dans un système de patriarcat renouvelé d'une part, et la promotion d'une piété féminine spécifique d'autre part - nous servira de fil conducteur pour examiner la condition des femmes dans le protestantisme français.

\section{La discipline du mariage chez les réformés}

13 Les protestants, en France comme ailleurs, ont rejeté la vie monastique et le célibat du clergé, qu'il s'agisse des prêtres, des moines ou des religieuses. Si les moines étaient dénoncés alors pour leur incontinence sexuelle, les religieuses, pensait-on, avaient besoin d'autant de discipline que les frères. Bien que les délits sexuels des nonnes fussent moins 
évidents, elles restaient des femmes soupçonnées d'être incapables de maîtriser leur sexualité.

Pour imposer une discipline sexuelle aux femmes, les réformateurs français ont commencé par s'intéresser à la famille - la première cellule sociale - et au lien du mariage. L'interprétation calviniste de la famille a accordé au mari et au père, sans aucun doute, une conscience exagérée de son rôle patriarcal. Le poids de l'Écriture sainte semblait renforcer la position du mari au sein de la famille. Saint Paul n'enseignait-il pas : "que les femmes soient soumises à leurs maris, comme au Seigneur " et "vous, enfants, obéissez à vos pères et à vos mères, en ce qui est selon le Seigneur... ». Le Décalogue n'exigeait-il pas : « Honore ton père et ta mère ${ }^{7}$ ». Jean Calvin et ses disciples, en parfaite connaissance des commandements bibliques, comparaient la famille à « une petite Église privée »: le père pieux, chef et maître de la famille, la supervise et la dirige conformément aux talents et aux capacités qu'il a reçus de Dieu's . Le père, l'ancien et sacré paterfamilias, assumait ainsi un rôle sacerdotal dans sa famille. De la même façon qu'il dirigeait les autres aspects de la vie familiale, le père était désigné comme un guide spirituel et moral. Dans la noblesse et la bourgeoisie cultivée, il officiait pour la maisonnée, l'épouse, les enfants et les serviteurs, lisant chaque soir l'Écriture sainte, conduisant les prières et chantant les psaumes. Les femmes, bien sûr, participaient avec leur mari à ces activités. Leur place au sein de la famille, en matière de pratique et de maintien des traditions religieuses, ne doit pas être négligée, surtout lorsque les Églises réformées de France furent attaquées par la majorité catholique.

Ce faisant, la Réforme a renforcé le pouvoir ecclésiastique sur le mariage et également, par extension, celui de l'État. Les protestants ont ainsi insisté sur l'indissolubilité des paroles de futur, c'est-à-dire des promesses d'un prochain mariage. Par ce moyen, ils ont essayé d'éviter des distinctions, qu'ils ont trouvé trop subtiles et parfois peu claires entre les paroles de futur (les fiançailles) et les paroles de présent (la cérémonie nuptiale au temple). Les futurs époux devaient aussi obtenir l'autorisation parentale, jusqu'à vingtcinq ans pour une femme et trente ans pour un homme. Le mariage était en effet d'abord une alliance entre deux familles, et les exigences de celles-ci prenaient le pas sur les vœux des individus. Les bans, publiés du haut de la chaire trois dimanches consécutifs, informaient la communauté. Après la bénédiction publiquement donnée par le pasteur, le mariage était inscrit dans le registre des mariages, pour éviter toute possibilité de contestation. Le divorce était possible, mais restait difficile à obtenir. Les causes en étaient limitées aux motifs les plus graves : l'adultère et l'absence prolongée. En réalité, l'homme apportait au consistoire une sentence judiciaire condamnant sa femme pour adultère, plus souvent que la femme ne s'y plaignait d'une longue absence de son mari'.

Toute une série de délits réprimandés par le consistoire est liée à des écarts de conduite commis à l'occasion du mariage - des fautes telles qu'un délai trop long entre les fiançailles et la célébration du mariage, ou même, parfois le refus apparent de faire célébrer le mariage après l'échange de promesses de fiançailles. Dans d'autres cas, des fiancés commençaient une vie commune avant la célébration du mariage, ou négligeaient de se procurer une permission écrite (et pas seulement orale) de leurs parents respectifs. Le plus souvent, le consistoire a pu venir à bout des difficultés et remettre les fautifs dans le droit chemin.

17 L'Église réformée, par le moyen du consistoire, a conforté l'autorité du père sur les enfants, et celle du mari sur sa femme. À Nîmes en 1581, Catherine Formentine s'opposait au mariage que son père avait arrangé entre elle et un capitaine Serre. La jeune fille 
prétendait qu'elle avait été fiancée avec Serre " par force ». Le consistoire interrogea tous les gens concernés et finalement, il excommunia la jeune femme pour son " opiniâtreté ». À Nîmes encore en septembre 1580, une jeune femme tenta de rompre les promesses de mariage qu'elle avait échangées avec un orfèvre. Évidemment elle éprouvait quelques regrets, non sans raison. Après que le consistoire ait exigé d'elle qu'elle se marie avec son fiancé (les paroles de futur étant indissolubles), le mari est retourné au consistoire trois mois plus tard, et il apparut alors qu'il s'était mis à battre sa femme ${ }^{10}$.

Une fois le mariage conclu, les consistoires essayèrent avec moins de succès de pacifier les rapports conjugaux. La violence domestique a en effet continué, même dans les maisons des anciens, malgré les admonitions consistoriales. Les anciens de Nîmes, assemblés trois ou quatre fois l'an dans des séances de censure réciproque, s'admonestaient « de ne pas battre » leurs femmes. Et à Saint-Gervais dans les Cévennes, où l'on rencontre un seul cas d'intervention du consistoire dans une dispute conjugale, ce fût pour exhorter un mari à ne pas traiter sa femme « inhumainement ${ }^{11} »$.

\section{Le contrôle de la conduite des femmes}

19 Les Églises réformées s'intéressaient plus à réprimer les rapports sexuels pratiqués hors mariage. Les tentatives d'élimination de la prostitution, de la fornication, de l'adultère et du concubinage étaient continuelles, et la plupart du temps elles visaient des femmes. Les délits sexuels et certaines autres fautes de comportement étaient du point de vue du consistoire essentiellement féminins. Si les femmes ne comptèrent que pour un tiers des délinquants convoqués par le consistoire de Nîmes au XVI ${ }^{\mathrm{e}}$ siècle, elles représentèrent les deux tiers des personnes accusées des péchés sexuels ou de paillardise - un mot qui désigne aussi bien l'adultère que la fornication ${ }^{12}$. Il semble bien que pasteurs et anciens partageaient les idées communes à l'encontre des femmes. Elles étaient perçues comme moins disciplinées et moins sensées que les hommes, et donc étaient incapables de maitriser leurs désirs sexuels. Sa faiblesse faisait de la femme une séductrice tentatrice et agressive en même temps qu'un « vaisseau fort fragile ${ }^{13}$ ".

Ce qui attirait immanquablement l'attention du consistoire sur la délinquance sexuelle, c'était la grossesse d'une femme non mariée. La grossesse - un signe évident et public des relations sexuelles - était la preuve de l'écart de conduite d'une femme célibataire. Victime classique de ce drame, la jeune femme employée comme domestique ou servante. Plus de la moitié des femmes enceintes convoquées par le consistoire de Nîmes se trouvaient dans cette situation. Un grand nombre avait émigré de la campagne vers la ville où elles ne purent résister aux avances du maître de la maison ou d'un autre serviteur. Marguerite Saurine, une jeune mère célibataire, a confessé au consistoire que son maître était le père de son enfant. Il était tombé malade et elle était obligé de dormir dans sa chambre pour veiller sur lui. Quand il guérit, elle se trouva enceinte. Les récits que les femmes font au consistoire ont toujours quelque chose de poignant. Ainsi, le 2 juillet 1578, les anciens de Nîmes interrogèrent-ils la chambrière Isabel Bonete :

« Interrogée à qui apartient l'enfant qu'elle a fait?

À répondu que l'enfant appartient au fils de Sire Vienne.

Interrogée à qui appartient l'enfant qu'[elle] avait fait devant cestuy ici ?

A répondu qu'[il] appartient au fils de Maître Pierre Rozel.

De rechef interrogée de qui étaient les autres enfants qu'elle avait fait devant ces deux? 
Elle se prosternait à terre, disant qu'elle était la plus grande pêcheresse qui jamais

aye été au monde et qu'elle demandait pardon à Dieu ${ }^{14}$ ".

21 D'autres formes du contrôle du comportement des femmes paraissaient plus légers. La fragilité et la séduction féminines s'exprimaient, pensait-on, dans la vanité des apparences : essentiellement les vêtements "dissolus", le maquillage et les coiffures immodestes. Les tenues vestimentaires jugées impudiques, destinées à attirer les regards et inciter à paillardise, sont peut-être les plus surveillées. Il s'agissait tout d'abord des décolletés ou «ouvertures de poitrine». Le consistoire condamnait également les artifices vestimentaires tels que les « hausseculs » ou « cache-bâtards ». Le fard, souvent vendu par les apothicaires, paraissait moins répréhensible mais restait pourtant condamné par les surveillants de la « modestie $»^{15}$.

La coiffure féminine a suscité davantage de discussion. Les consistoires s'élevaient en effet contre les entortillements de cheveux, remonté en forme de cornes au moyen d'armatures d'airain. Plus que la forme ou l'agencement ainsi condamnés, l'arrangement de cheveux avait pour les femmes une importance sociale et symbolique. Au moment du mariage, la femme dissimulait ses cheveux. Les cheveux longs et flottants étaient le symbole d'une femme disponible: la prostituée pour la paillardise ou la vierge pour le mariage. Ce n'était pas là simplement une question de respect, il fallait aussi que les femmes réduisent leur attrait sexuel. De plus, le Nouveau Testament et les anciens auteurs chrétiens tel que Tertullien ont ordonné aux femmes de couvrir leur tête. Les pasteurs revendiquaient donc l'autorité de la Sainte Bible et de l'Église ancienne à l'appui de leur position. La question de fond était celle de la discipline sexuelle de la femme et du maintien d'un ordre moral dans lequel elle était subordonnée à l'homme ${ }^{16}$.

\section{Une reconnaissance des compétences religieuses de la femme?}

Outre le contrôle de la sexualité de la femme, il y avait celui de ses compétences et capacités spirituelles. Le consistoire calviniste a proscrit les rôles sacrés attribués aux femmes dans la religion populaire du Moyen-Âge. Il s'agissait souvent de pratiques magiques. Le consistoire se plaignait des «devines» et sorcières qui pratiquaient la médecine populaire et utilisaient des plantes et des formules magiques. Une «femme devineresse » de Layrac, par exemple, avait ainsi guéri la fille de Noémi Fornol. Elle fut vivement censurée parce que "c'est de la main de Dieu que nous devons attendre le secours et guérison de nos maux $»^{17}$. On consultait aussi des " devines » et bohémiennes pour connaître l'avenir ou dans l'espoir de retrouver un objet personnel ou une somme d'argent perdus.

Le consistoire tentait également de supprimer certaines traditions ancestrales et féminines destinées à protéger les nouveau-nés. Les pasteurs et anciens ont donc sanctionné sévèrement des sage-femmes et des voisines qui baptisaient les enfants au moment de leur naissance. Le rejet réformé de la croyance médiévale aux limbes a supprimé la nécessité de l'ondoiement des nouveau-nés fragiles. Mais du même coup, on éliminait un rôle rituel et spirituel important jusque-là reconnu aux femmes.

Les dimensions de ces pratiques féminines et de la réponse protestante sont bien illustrées par un incident qui se situe à Ganges vers la fin du XVI ${ }^{\mathrm{e}}$ siècle ${ }^{18}$. Il y est question d'une sorte de pèlerinage accompli par trois femmes au printemps 1597. Le trio avait fait 
appel au pouvoir du protecteur médiéval de la ville pour guérir un enfant tombé gravement malade.

Un mardi matin du mois d'avril donc, ces trois pauvres femmes vinrent à Ganges. Descendues du hameau cévenol de Saint-Roman-de-Codières, elles portaient dans leurs bras un enfant malade. Selon le consistoire elles l'avaient "voué à quelque idole ", en l'occurrence Saint-Pierre. Arrivées à Ganges, les trois femmes s'arrêtèrent à l'Hôtel-Dieu où il semble qu'elles demandèrent une assistance financière. Ensuite, elles cherchèrent Donne Figuieyresse dite La Sauvage. Puis le groupe se rendit au cimetière " pour faire les superstitions et idolâtries ». L'hospitalier leur avait dit que Jacquette Vincente, dont la maison touchait au cimetière, les laisserait passer par sa porte pour y entrer. En passant par la maison de Vincente, elles posèrent « une pièce de pain sur le tison du feu, luy disant qu'il était pour quelque chien ou chat ». Ayant pris du feu chez Vincente, elles s'en allèrent alors à l'endroit du cimetière que l'on appelait « derrière Saint-Pierre ", (c'est-àdire, derrière l'ancienne église Saint-Pierre). Accompagnées de Donne Figuieyresse, elles $\mathrm{y}$ allumèrent neuf chandelles et firent « leurs superstitions et offrandes à Saint-Pierre ». Enfin, les trois femmes donnèrent quelques pièces d'argent à Donne Figuieyresse avant de disparaître dans la montagne.

Le consistoire de Ganges, réuni deux jours après, appela Jacquette Vincente et Donne Figuieyresse. Vincente comparut le dimanche matin pour expliquer son rôle dans les événements. Le pasteur et les anciens la censurèrent parce qu'au lieu de « détourner » les trois pauvres femmes de "ces idôlatries, elles les a assistées et aidées ». Vincente a alors « demandé pardon à Dieu et a promis de n'y retourner plus ». La Sauvage ne se présenta pas au consistoire. Peut-être n'était-elle pas protestante et donc avait moins à craindre de la sévérité des hommes de l'Église réformée. Il est également probable que La Sauvage avait déjà été marginalisée par les responsables de la communauté et qu'elle pensait qu'il n'y avait plus lieu de respecter l'ordre des hommes qui, de toute façon, n'auraient pas toléré son comportement.

Certes, les divers éléments de cette histoire méritent attention: la juxtaposition des traditions chrétiennes et non chrétiennes dans le symbolisme et le rituel, l'angoisse maternelle face à un enfant mourant, l'offrande du pain et l'importance du feu, l'emploi des chandelles et le don de l'argent, le choix du cimetière pour des cérémonies thaumaturges, la vitesse et l'intensité de la réaction consistoriale. Cependant je voudrais ici privilégier l'importance du rôle médiateur de La Sauvage.

«L'espace » féminin qu'ouvrait la religion populaire était très large. En mars 1599, une autre gangeoise avait déjà conseillé à sa voisine de « promettre une sienne fille qui était malade à certaines idoles et leur donner certaines chandelles et argent ${ }^{19}$ ». Évidemment les prières aux saints, les chandelles et l'offrande de l'argent - des actes personnels et, il semble, fortement féminins - donnaient de l'espoir et de la sécurité au peuple. Par contre, une catastrophe pouvait amener un homme ou une femme à se retourner contre les autres membres de la communauté et parfois à s'approprier les symboles du pouvoir religieux des femmes. Ainsi un couple de Saint-Amans, à la mort de l'un de ses enfants, fut-il conduit à s'en prendre à la belle-sœur. Elle était, selon les parents de l'enfant décédé, une sorcière qui avait « donné d'argent pour faire dire des messes et avait donné neuf pains d'offrande tous chauds sortant du four ». Par ce moyen, elle a « tué leur enfant $20 »$.

L'interprétation par le consistoire des événements concernant La Sauvage et les trois femmes cévenoles était faussée par son parti pris antiféminin et son sens de la hiérarchie. 
Il s'agissait de femmes pauvres et étrangères. Ces femmes avaient besoin de surveillance et ces "pauvres étrangères" paraissaient menaçantes. Même la présence de l'unique participante gangeoise, Donne Figuieyresse dite La Sauvage, semble avoir été ressentie comme une présence extérieure et troublante. Bien qu'on n'ait jamais prononcé le mot sorcière, La Sauvage était une personne déconcertante et ambiguë aux yeux des anciens. Son surnom, La Sauvage, fait allusion à quelqu'une qui vivait aux marges de la société. Par contre, l'emploi du titre "Donne» exprime du respect - pour l'âge, le statut, ou la fonction - de la part de certains membres de la communauté. Et c'est elle qui a présidé aux rites qui eurent lieu au cimetière. D'une certaine manière, l'incident souligne le rôle sacré attribué à certaines femmes, cela précisément que les protestants voulaient supprimer.

\section{Les traits positifs d'une évolution}

31 Les aspects positifs de la condition des femmes protestantes, quoiqu'ils soient moins évidents, sont aussi considérables que les éléments négatifs, soumis à répression. La contribution de l'épouse et de la mère au bien-être de la famille était immense mais discrète, car elle s'exerçait davantage dans la sphère privée que dans la sphère publique réservée au mari. Son rôle restait pourtant essentiel. Après la naissance des enfants, la femme s'occupait en effet de les élever et de les instruire pendant les six ou sept premières années de leur vie. Le devoir maternel et domestique s'étendait jusqu'à la surveillance quotidienne de l'éducation morale et religieuse des enfants. Thomas Lambert, qui vient de soutenir une thèse sur la discipline sociale et morale à Genève au $\mathrm{XVI}^{\mathrm{e}}$ siècle - une thèse fondée sur les registres de délibérations du consistoire au temps de Calvin - a démontré que la mère genevoise était responsable de l'introduction des enfants dans la vie religieuse réformée, en ce sens qu'elle avait charge de leur inculquer la nouvelle manière de prier en apprenant par cœur, par exemple, le Notre Père et le Symbole des Apôtres en français ${ }^{21}$.

Après la révocation de l'Édit de Nantes, le rôle décisif des mères et des grand-mères dans l'éducation des enfants a pris une importance accrue. La responsabilité traditionnelle confiée à la mère en matière de formation religieuse des enfants a contribué énormément à la conservation de la tradition réformée en France. Les efforts maternels comptèrent parmi les moyens principaux de la transmission de la foi protestante après 1685 , puisque la pratique religieuse s'est alors déplacée du domaine public au privé, de l'extérieur à l'intérieur, du masculin au féminin.

Il y avait au moment de la Révocation des divisions religieuses dans les familles protestantes. Le plus souvent, il s'agissait de ruptures entre parents et enfants, ou au sein de la fratrie. Une autre sorte de division, peut-être plus révélatrice, opposait les femmes aux maris. La réaction des conseillers (ou juges) protestants à la chambre de l'Édit de Castres, un groupe sur lequel j'ai fait des recherches approfondies, en donne de nombreux exemples $^{22}$. En 1685, les conseillers protestants eurent trois mois pour se convertir ou démissionner de leurs offices. Et ils se sont tous conformés à la volonté royale. Thomas d'Escorbiac, un conseiller huguenot d'origine montalbanaise, constatant qu'il ne se trouvait plus «un seul huguenot dans tout Montpellier, ni dans Nîmes, non plus qu'ailleurs ", se rendit à Versailles pour abjurer devant le Roi-Soleil lui-même. En retour, Escorbiac retint son office judiciaire et quelques années plus tard il résigna sa charge en faveur de son fils aîné. Mais les trois autres fils restèrent protestants, et émigrèrent aux 
Pays-Bas et au Danemark. Sa femme se montra également plus obstinée, mais, en fin de compte, elle se convertit ${ }^{23}$.

Un autre conseiller huguenot, Jacques de Lacger abjura sa foi d'une façon moins dramatique. Pourtant la division religieuse entre lui et sa femme, Magdelaine de Falguerolles, resta permanente. Pour garder son office de juge, il se convertit en septembre 1685. Mais sa femme refusa de devenir catholique, probablement avec l'accord explicite de son mari. Sans doute, elle souhaitait rester, sans bruit et en privé, l'instrument familial de la conservation des croyances et des pratiques protestantes. Une telle stratégie paraît en accord avec le rôle primordial des femmes dans l'éducation religieuse des enfants. L'accord familial sur les éléments essentiels de cette stratégie est confirmé par le fait que Jacques de Lacger a adressé une pétition à la monarchie en demandant que l'on pardonne à sa femme un tel refus de conversion ${ }^{24}$.

Leur stratagème, bien conçu pour une courte durée (qui aurait pensé que la Révocation était définitive ?) a échoué au moment de la mort de Jacques de Lacger en 1688. Bien qu'il fût inhumé dans la cathédrale Saint-Étienne à Toulouse, les officiers du roi soumirent alors la veuve à une pression implacable dans le but de la convertir au catholicisme. On alla jusqu'à menacer Magdelaine de Falguerolles et sa sœur Blanche, la femme d'un autre conseiller qui était "nouveau catholique", de les bannir en Bourgogne. Il semble que Magdelaine fut emprisonnée quelques mois à Albi en automne 1699. Mais sa fermeté religieuse ne fut jamais ébranlée.

D'autres couples ont tenté d'user de la même tactique : le mari abjurait afin de conserver son office tandis que la femme et, avec un peu de chance, les enfants, restaient protestants. Blanche de Falguerolles, la sœur de Magdelaine, et son mari le conseiller Salomon de Faure ont agi ainsi. Il est devenu catholique; elle est restée obstinément réformée. La force de l'attachement religieux de ces femmes est sans ambiguïté. En 1700, le Parlement de Toulouse condamna in absentia Dame Daubiais, femme du conseiller Claude de Juge, un autre "nouveau catholique». Évidemment son effort pour rester protestante fut particulièrement coûteux puisqu'elle avait déjà du quitter le royaume ${ }^{25}$.

Barbara Diefendorf, dans une étude des divisions religieuses dans les familles de la France septentrionale au $\mathrm{XVI}^{\mathrm{e}}$ siècle, présente un autre aspect des divergences religieuses entre femme et mari ${ }^{26}$. Plutôt que d'une décision mutuelle et délibérée prise par le couple, il s'agissait là d'une véritable rupture confessionnelle. C'est dans le cadre de l'anarchie des guerres de religion qu'elle examine les tensions qui provenaient des différences religieuses et les moyens par lesquels les familles ont fait face à des situations déchirantes. Vue la tradition de domination du mari sur la femme, il peut sembler surprenant que les femmes aient pu différer de leurs maris sur une question aussi importante que celle de la religion. Il est évident, néanmoins, que certaines d'entre elles nourrirent des opinions indépendantes quant à leur foi.

De nombreux désaccords religieux ont surgi quand la femme est devenue protestante alors que son mari restait catholique. Dans le cas d'un mariage mixte, les catholiques reprochaient aux protestants la destruction des liens traditionnels de solidarité familiale. Mais Calvin avait déjà conseillé aux femmes dont les maris restaient catholiques de rester à leur côté et d'essayer de les gagner à la vérité. Même si elle ne réussissait pas à le convertir, la femme ne devait pas quitter son mari. L'obligation de soumission à l'autorité maritale n'était levée qu'au moment où la vie - ou l'immortalité de l'âme - était mise en péril. 

Normandie avec les listes des personnes qui ont préféré fuir plutôt que se convertir ${ }^{27}$. Par ce moyen, elle a identifié ce qui semble être une forte proportion des femmes qui sont restées protestantes alors que leurs maris sont retournés à la foi catholique. C'est la preuve, selon Diefendorf, de l'indépendance et du courage des femmes protestantes face au danger, face au mari.

40 à bien des égards. Malgré le renforcement continuel de l'idée de soumission et d'obéissance de la femme à l'autorité du mari, certaines femmes protestantes ont trouvé les moyens de se moquer de leurs maris, pères et frères - tous des hommes qui ont voulu les mettre sous discipline. Ainsi la veuve d'un chaussetier de Montauban, tout en exagérant bien entendu, fit remarquer que les hommes qui «ne pouvaient avoir des enfants et qui n'embrassaient leurs femmes six ou sept fois la nuit méritaient d'être menés à la place et y empalés $\aleph^{28}$. Bien que la veuve ait tout nié, les anciens et les consuls - l'élite patriarcale - furent extrêmement contrariés de ce défi féminin. Dans les paroles de la femme, le phallus - image et symbole de l'autorité et du pouvoir masculin - a failli à sa réputation et, par conséquent, il est devenu l'instrument du châtiment !

\section{Éléments de conclusion}

41

Les éléments du portrait de la femme protestante aux XVI et XVII siècles sont divers et complexes, complémentaires et parfois contradictoires. Les hommes qui ont dirigé la Réforme ont cherché à renforcer une hiérarchie déjà très fortement établie. Les Églises réformées et leurs consistoires ont affirmé le pouvoir du mari dans le mariage et la famille. Ils ont, dans le même temps, établi des procédures de contrôle de la sexualité des femmes. En outre, les consistoires ont réduit la sphère féminine d'influence spirituelle et religieuse. Ces efforts pour discipliner la femme et la placer sous l'autorité masculine furent largement suivis d'effets. Mais il semble que les femmes ont aussi trouvé dans le protestantisme les moyens de créer leur propre « espace ». Ainsi la mère protestante futelle dès le début responsable de l'éducation morale et religieuse des enfants. L'ironie, c'est que, après la Révocation, la femme, qui était jusque-là placée en retrait au sein de la famille, devint l'actrice irremplaçable de la transmission de la foi. Avec la proscription de la pratique publique du protestantisme qui relevait du monde masculin, la sphère privée et féminine acquit une importance prépondérante. Mais, on l'a vu, la femme protestante avait toujours su garder la capacité de se comporter avec force et indépendance.

\section{BIBLIOGRAPHIE}

La plupart des « registres de consistoires » sont conservés à l'état de manuscrits. On peut les consulter, selon les cas, dans des Archives municipales ou départementales, aux Archives Nationales, à la Bibliothèque Nationale de France, ou encore à la Bibliothèque de la Société de

Archives de sciences sociales des religions, 113 | janvier-mars 2001 
l'Histoire du Protestantisme Français. Certains registres ont été, totalement ou partiellement, publiés.

BAINTON Roland, Women of the Reformation in Germany and Italy, Minneapolis, Augsburg Publishing House, 1971.

BELS Pierre, Le mariage des protestants français jusqu'en 1685, Paris, Librairie Générale de Droit et de Jurisprudence, 1968.

CHAREYRE Philippe, “'The Great Difficulties One Must Bear to Follow Jesus Christ': Morality at Sixteenth-Century Nîmes", in Raymond A. MENTZER, éd., Sin and the Calvinists: Morals Control and the Consistory in the Reformed Tradition, Kirksville, Sixteenth Century Journal Publishers, 1994, pp. 63-96.

DAVIS Natalie Z., Les cultures du peuple. Rituels, savoirs et résistances au XVI ${ }^{e}$ siècle, Paris, AubierMontaigne, 1979.

DIEFENDORF Barbara B., « Les divisions religieuses dans les familles parisiennes avant la SaintBarthélemy ", Histoire, économie et société, 7,1, 1988, pp. 55-77.

DIEFENDORF Barbara B., "Houses Divided : Religious Schism in Sixteenth-Century Parisian Families", in S. ZIMMERMAN, R. WEISSMAN, éds., Urban Life in the Renaissance, Newark, University of Delaware Press, 1989, pp. 80-99.

GARRISSON-ESTEBE Janine, L'Homme protestant, Paris, Hachette, 1980.

GARRISSON Janine, Les Protestants au XVI ${ }^{e}$ siècle, Paris, Fayard, 1988.

KINGDON Robert M., Adultery and Divorce in Calvin's Geneva, Cambridge (MA), Harvard University Press. 1995.

LAMBERT Thomas, Preaching, Praying, and the Reform in Sixteenth-Century Geneva, thèse de doctorat, Université de Wisconsin, 1998.

MENTZER Raymond A., "Disciplina nervus ecclesiae: The Calvinist Reform of Morals at Nîmes", Sixteenth Century Journal, 18, 1987, pp. 89-115.

MENTZER Raymond A., Blood and Belief: Family Survival and Confessional Identity among the Provincial Huguenot Nobility, West Lafayette, Purdue University Press, 1994.

MENTZER Raymond A., “The Persistence of 'Superstition and Idolatry' among Rural French Calvinists”, Church History, 65, 1996, pp. 220-233.

OZMENT Steven, When Fathers Ruled: Family Life in Reformation Europe, Cambridge (MA), Harvard University Press, 1983.

ROPER Lyndal, The Holy Household: Women and Morals in Reformation Augsburg, Oxford, Oxford University Press, 1989.

WIESNER Merry E., Women and Gender in Early Modern Europe, Cambridge, Cambridge University Press, 1993.

\section{NOTES}

1. KINGDON, 1995, p. 4.

2. Voir, par exemple Roland BAINTON, 1971, et plus récemment, Steven OzMENT, 1983. 
3. Natalie Z. DAVIS, «Les huguenotes,» in : Natalie DAVIS, 1979, pp.113-158; [trad. de "City Women and Religious Change" in: Dorothy McGuigan, éd., A Sampler of Women's Studies, Ann Arbor, University of Michigan, Center for Continuing Education of Women, 1973].

4. Jean CRESPIN et Simon GoULART, Histoire des Martyrs persecutez et mis à mort pour la verité de l'Evangile depuis le temps des Apostres jusques à present (1619) (édité pour la première fois en 1554), Daniel Benoît, éd., 3 t., Toulouse, Société des Livres Religieux, 1885-1889 (édition du texte de 1619).

5. ROPER, 1989.

6. Voir, par exemple. Janine GARRISSON, 1988.

7. Éphésiens 5, 22 et 6,1; Exode 20, 12 .

8. GARRISSON, op. cit., pp. 37-39, 88-91.

9. La doctrine et la pratique juridique du mariage chez les protestants français ont été étudiées par Pierre BELS, 1968.

10. Bibliothèque Nationale (dorénavant B.N.), ms. fr. 8667 , Registre du Consistoire de Nîmes (1578-1584), fols. 141, 152, 156, I57,160v, 187, 190v, 194v, 290, 333, 366v, 372-372v.

11. Archives Nationales, TT 269, 25, Registre du Consistoire de Saint-Gervais (1564-1568), fol. 964. B.N., ms. fr. 8666, Registre du Consistoire de Nîmes (1561-1562), fols, 1v, 18, 32-32v, 62-64, $105-105 \mathrm{v}$

12. MentZer, 1987, pp. 103, 110-111.

13. Voir consistoire de Montauban, Archives Départementales (dorénavant A.D.), Tarn-etGaronne, I 1, Registre du Consistoire de Montauban (1595-1598) fols. 113v-114 (1596) ; cf. 1 Pierre 3, $7:$ «...vivez sagement avec vos femmes, les traitant avec honneur et avec discrétion, comme le sexe le plus faible...)

14. B.N., ms. fr. 8667 , fols. $4,5,6.19 v$.

15. CHAREYRE, 1994, pp. 85-87.

16. WIESNER, 1993, pp. 179-180.

17. A.D., Gers, 23067 (H 77, Hôpital de Condom), Registre du Consistoire de Layrac (1578-1634), le 17 août 1618.

18. A.D., Hérault, E Dépôt, Ganges GG 24, Registre du Consistoire de Ganges (1587-1609), fols. 80-82 ; MENTZER, 1996, pp. 220-233.

19. A.D., Hérault. E Dépôt, Ganges GG 24, fol. 101.

20. A.D., Tarn, I 8. Registre du Consistoire de Saint-Amans (1587-1604). le 30 mars, le 2 et le 3 avril 1597.

21. LAMBERT, 1998.

22. MENTZER, 1994.

23. GARRISSON-ESTEBE, 1980, pp. 58-61.

24. A.D., Haute-Garonne, B 1884, fol. 216; Archives de la famille de Falguerolles, dossiers généalogiques ; Bibliothèque de la Société de l'Histoire du Protestantisme Français, ms. G, dossier Romane-Musculus, no. 64.

25. A.D., Haute-Garonne, B 1227, fol. 284 ; Archives de la famille de Falguerolles, dossiers généalogiques.

26. DiEFENDORF, 1988 , pp. 55-77.

27. DIEFENDORF, 1989, pp. 80-99.

28. A.D., Tarn-et-Garonne. I 8, fols. 227, 230-230v, 235. 


\section{RÉSUMÉS}

La diversité des informations concernant la condition de la femme protestante aux $\mathrm{XVI}^{\mathrm{e}}$ et $\mathrm{XVII}{ }^{\mathrm{e}}$ siècles explique que leur interprétation est complexe. Des historiens ont commencé par accentuer les effets positifs de la Réforme sur la condition féminine. Ensuite, les études ont conduit à des conclusions plus nuancées. Les hommes qui ont dirigé la Réforme paraissent en effet avoir contribué à renforcer le patriarcat familial et une hiérarchie sociale très fortement attestée. Nos recherches, qui ont porté sur un grand nombre de registres de consistoires des Églises locales, conduisent à penser que les efforts des Églises réformées et de leurs consistoires pour placer la femme sous l'autorité masculine furent largement suivis d'effets. Mais il apparaît aussi, que du fait des conditions d'existence des protestants français, les femmes ont trouvé à créer, puis à garder pour elles-mêmes un « espace » dans lequel elles tinrent un rôle de premier plan. Les nouvelles conceptions du mariage et certains usages ecclésiastiques ont en effet permis aux femmes réformées de conserver la capacité de se comporter avec une force et une indépendance qui, souvent, renvoie à des situations antérieures à la Réforme.

The various interpretations surrounding the condition of Protestant women during the sixteenth and seventeenth centuries are diverse and complex. Historians initially emphasized the positive aspects of the Reformation on the role and status of women. Subsequent studies have been far more cautious in their conclusions. The men who led the Reformation seem to have worked for the reinforcement of patriarchy and the strengthening of a rigid social hierarchy. My own research, which is based on the examination of a substantial number of consistory registers from local churches, suggests that the efforts of the French Reformed Churches and their consistories were vigorous and effective. But it also appears that many Protestant women were able to establish and maintain their own feminine "space," one which they dominated. The Protestant reconceptualization of marriage and certain ecclesiastical practices, for example, allo-wed Protestant women to retain the capacity to act with a force and independence that often recalled the situation prior to the Reformation.

La diversidad de las informaciones sobre la condición de la mujer protestante en los siglos XVI y XVII iniplica una interpretación compleja. Distintos historiadores empezaron resaltando los efectos positivos de la Reforma sobre la condición feminina. Después, los estudios llegaron a conclusiones más matizadas. En efecto, parece que los hombres que condujeron la Reforma contribuyeron a fortalecer el pratiarcado familiar y una jerarquía social muy marcada. A partir del estudio de numerosos registros de consistorios de iglesias locales, nuestras investigaciones permiten pensar que los esfuerzos de las Iglesias reformadas y de sus consistorios para colocar a la mujer bajo la autoridad. masculina tuvieron muchos efectos en la realidad. Sin embargoo, se desprende también de nuestro estudio que, por las condiciones de vida de los protestantes franceses, las mujeres pudieron crear y conservar un « espaclo » propio donde tuvîeron un papel de primer importancia. En efecto, las nuevas concepciones del matrimonio y algunos usos eclesiásticos permitieron a las mujeres reformadas mantener la capacidad de comportarse cou una fuerza y una independencia que, frecuentemente, es comparable a situaciones anteriores a la Reforma. 
AUTEUR

RAYMOND A. MENTZER

Montana State University 